\title{
Vanilloid-Sensitive Afferents Activate Neurons with Prominent A-Type Potassium Currents in Nucleus Tractus Solitarius
}

\author{
Timothy W. Bailey, Young-Ho Jin, Mark W. Doyle, and Michael C. Andresen \\ Department of Physiology and Pharmacology, Oregon Health and Science University, Portland, Oregon 97201-3098
}

Cranial visceral afferents innervate second-order nucleus tractus solitarius (NTS) neurons via myelinated (A-type) and unmyelinated (C-type) axons in the solitary tract (ST). A- and C-type afferents often evoke reflexes with distinct performance differences, especially with regard to their frequency-dependent properties. In horizontal brainstem slices, we used the vanilloid receptor 1 agonist capsaicin (CAP; 100 nM) to identify CAPsensitive and CAP-resistant ST afferent pathways to secondorder NTS neurons and tested whether these two groups of neurons had similar intrinsic potassium currents. ST stimulation evoked monosynaptic EPSCs identified by minimal synaptic jitter $(<150 \mu \mathrm{sec})$ and divided into two groups: CAP-sensitive $(n=37)$ and CAP-resistant $(n=22)$. EPSCs in CAP-sensitive neurons had longer latencies $(5.1 \pm 0.3$ vs $3.6 \pm 0.3 \mathrm{msec} ; p=$ 0.001 ) but similar jitter ( $p=0.57$ ) compared with CAP-resistant neurons, respectively. Transient outward currents (TOCs) were significantly greater in CAP-sensitive than in CAP-resistant neurons. Steady-state currents were similar in both groups. 4-Aminopyridine or depolarized conditioning blocked the TOC, but tetraethylammonium had no effect. Voltage-dependent activation and inactivation of TOC were consistent with an A-type $\mathrm{K}^{+}$current, $I_{\mathrm{KA}}$. In current clamp, the activation of $I_{\mathrm{KA}}$ reduced neuronal excitability and action potential responses to ST transmission. Our results suggest that the potassium-channel differences of second-order NTS neurons contribute to the differential processing of A- and C-type cranial visceral afferents beginning as early as this first central neuron. $I_{\mathrm{KA}}$ can act as a frequency transmission filter and may represent a key target for the modulation of temporal processing of reflex responsiveness such as within the baroreflex arc.

Key words: sensory; vanilloid; glutamate; presynaptic modulation; autonomic; visceral; baroreceptor; baroreflex
Cranial afferent information is conveyed by a mixture of myelinated (A-type) and unmyelinated (C-type) axons that form the solitary tract (ST) to synapse in the nucleus tractus solitarius (NTS). These two afferent classes appear to have important functional differences in their contributions to homeostatic regulatory reflexes (Loewy, 1990; Andresen and Kunze, 1994). In particular for example, A-type arterial baroreceptors have lightly myelinated axons, higher conduction velocities, and lower pressure thresholds compared with C-type baroreceptors (Kunze and Andresen, 1991). The reflexes evoked by selective activation of Aand $\mathrm{C}$-type baroreceptor afferents can have quite different frequency-response characteristics (Fan and Andresen, 1998; Fan et al., 1999). Little is known about the mechanisms responsible for these differences in A- and C-type reflexes. Here we take advantage of the presence of the vanilloid receptor 1 (VR1) on C-type axons (Fan and Andresen, 1998; Doyle et al., 2002) and use capsaicin (CAP) to identify the general class of ST afferent input to second-order NTS neurons and examine the properties of the postsynaptic neurons contacted by these two classes of cranial visceral afferent CAP-sensitive and CAP-resistant neurons.

The cellular processes within and beyond the NTS that contribute to differential processing of A- and C-type cranial visceral afferents are unknown. Some of these autonomic pathways may

\footnotetext{
Received April 5, 2002; revised June 10, 2002; accepted June 13, 2002.

This work was supported by National Institutes of Health Grant HL-41119 and by grants from the National Center of the American Heart Association.

Correspondence should be addressed to Dr. Michael C. Andresen, Department of Physiology and Pharmacology, Oregon Health and Science University, Portland, OR 97201-3098. E-mail: andresen@ohsu.edu.

M. W. Doyle's present address: Department of Biology, George Fox University, Newberg, OR 97132-2697

Copyright (C) 2002 Society for Neuroscience $\quad 0270-6474 / 02 / 228230-08 \$ 15.00 / 0$
}

have as few as two central neurons (e.g., the cardiac parasympathetic pathway) (Standish et al., 1994). The NTS contains the initial synapse on the second-order neuron of these paths, but the second-order neurons are intermixed within a heterogeneous population with varying intrinsic properties. Previous studies suggested that the transient A-type voltage-gated $\mathrm{K}^{+}$current $I_{\mathrm{KA}}$ is one of the varied features of these NTS neurons (Dekin and Getting, 1984, 1987; Dekin et al., 1987; Moak and Kunze, 1993; Paton et al., 1993). The presence of $I_{\mathrm{KA}}$ affects the excitability and firing characteristics of neurons (Rudy, 1988; Malin and Nerbonne, 2001), but the relationship of these differences to the overall function of the nucleus is unknown. Here we report the unexpected finding that CAP can be used to distinguish two different groups of second-order neurons (Doyle et al., 2002) that differ in their expression of $\mathrm{K}^{+}$currents. The $I_{\mathrm{KA}}$ currents are larger in CAP-sensitive second-order NTS neurons; this difference in $I_{\mathrm{KA}}$ expression reduces overall excitability and the ability of ST activation to evoke action potentials. Thus, preferential expression of $I_{\mathrm{KA}}$ in NTS neurons with input from VR1containing afferents contributes to the differential processing of C-type cranial visceral afferents within the NTS.

\section{MATERIALS AND METHODS}

NTS slices. Horizontal slices of the hindbrain of male Sprague Dawley rats (150-350 gm; Charles River Laboratories, Wilmington, MA) were prepared as described previously (Doyle et al., 2002). All animal procedures were conducted with the approval of the University Animal Care and Use Committee in accordance with U.S. Public Health Service Policy on Humane Care and Use of Laboratory Animals and the National Institutes of Health Guide for the Care and Use of Laboratory Animals. Solutions, recording conditions, and the focus on medial portions of the NTS (mNTS) were as described previously (Doyle et al., 2002). 
Electrophysiological recordings. Patch recording electrodes (1.8-3.5 $\mathrm{M} \Omega$ ) were filled with a solution composed of (in $\mathrm{mM}$ ): $10 \mathrm{NaCl}, 130 \mathrm{~K}$ gluconate, 11 EGTA, $1 \mathrm{CaCl}_{2}$, and $2 \mathrm{MgCl}_{2}, 2 \mathrm{ATP}, 0.2 \mathrm{GTP}, 10$ HEPES, pH 7.3, 295 mOsm. Selection criteria were based on the recording stability and the monosynaptic jitter of ST EPSC latency (Doyle et al., 2002). In addition to voltage-clamp recordings (Axopatch 200A; Axon Instruments, Foster City, CA) described previously (Doyle et al., 2002), some experiments used single-electrode continuous voltage-clamp and current-clamp recordings in the same neurons (Axoclamp 2A; Axon Instruments).

For assessments of outward current responses, voltage-clamp protocols included command voltage steps used to evoke transient outward currents (TOCs) and steady-state currents (SSCs). The voltage dependence of the activation of these currents was assessed by initially conditioning at $-90 \mathrm{mV}(500 \mathrm{msec})$ followed by longer activation steps $(1200 \mathrm{msec})$ to voltages ranging from -100 to $0 \mathrm{mV}$. Voltage-dependent inactivation of TOCs was assessed with conditioning steps $(500 \mathrm{msec})$ ranging from -100 to $0 \mathrm{mV}$ that were followed by a longer test step $(1200 \mathrm{msec})$ to -10 $\mathrm{mV}$ (see Fig. 2). SSCs were measured near the end of the second long step in each protocol. The peak of the TOCs was measured after capacitive transients had subsided at 3-5 msec after the initiation of the long step and SSC subtracted. In some experiments, current-clamp recordings were used to assess the effect of activating TOCs on the firing properties of second-order neurons by injecting depolarizing and hyperpolarizing steps of current to determine the effect on current-evoked firing and ST-evoked responses (see Figs. 6 and 7). Tetraethylammonium (TEA) and 4-aminopyridine (4-AP) were obtained from Sigma (St. Louis, MO). Both TEA and 4-AP were dissolved in extracellular solution on the day of the experiment.

All data are presented as averages \pm SEM. Statistical comparisons were made using either unpaired Student's $t$ test, repeated-measures (RM) ANOVA, or one-way ANOVA followed by Fisher's PLSD post hoc analysis when appropriate (see individual results) (Statview 4.57; Abacus Concepts, Calabasas, CA). $p<0.05$ indicated a significant difference.

\section{RESULTS}

\section{CAP-sensitive ST-evoked synaptic responses}

The shock-to-shock variability of the EPSC response latencies to electrical activation identifies mNTS neurons monosynaptically connected to the ST in horizontal brainstem slices (Doyle and Andresen, 2001). We selected neurons with an EPSC jitter of $<150 \mu \mathrm{sec}$ for study as second-order neurons. Challenges with CAP (100 nM) divided these neurons into two groups on the basis of the CAP sensitivity of their EPSCs. ST-evoked EPSCs of CAP-sensitive mNTS neurons were blocked by sustained exposure to CAP (Fig. 1A, left). In the remainder of the mNTS second-order neurons, CAP did not alter their ST-evoked EPSCs (CAP-resistant) (Fig. 1 $\mathrm{A}$, right). Note that ongoing spontaneous EPSCs were not blocked in both classes of neurons. The STevoked EPSCs of CAP-sensitive neurons had a significantly longer latency on average $(5.1 \pm 0.3 \mathrm{msec} ; n=37)($ Fig. $1 B$, left $)$ compared with CAP-resistant mNTS neurons $(3.6 \pm 0.3 \mathrm{msec}$; $n=22 ; p=0.0016$; Student's $t$ test). However, latency alone poorly predicted EPSC sensitivity to CAP blockade, because the distribution of latencies for the two groups overlapped considerably (Fig. $1 B$, right). The average jitter for CAP-sensitive (82.0 \pm $4.8 \mu \mathrm{sec})$ and CAP-resistant $(86.9 \pm 7.6 \mu \mathrm{sec})$ neurons was not different ( $p=0.57$; unpaired Student's $t$ test).

\section{Prominent transient voltage-dependent outward currents in CAP-sensitive mNTS neurons}

The pattern of time- and voltage-dependent outward currents activated by depolarization differed significantly between CAPsensitive and CAP-resistant mNTS neurons (Fig. 2). From a potential of $-90 \mathrm{mV}$, sustained depolarization elicited large outward currents in CAP-sensitive neurons that decayed over time (Fig. 2A, top). Conditioning such neurons at progressively more depolarized potentials virtually eliminated these early tran-
A CAP-Sensitive CAP-Resistant

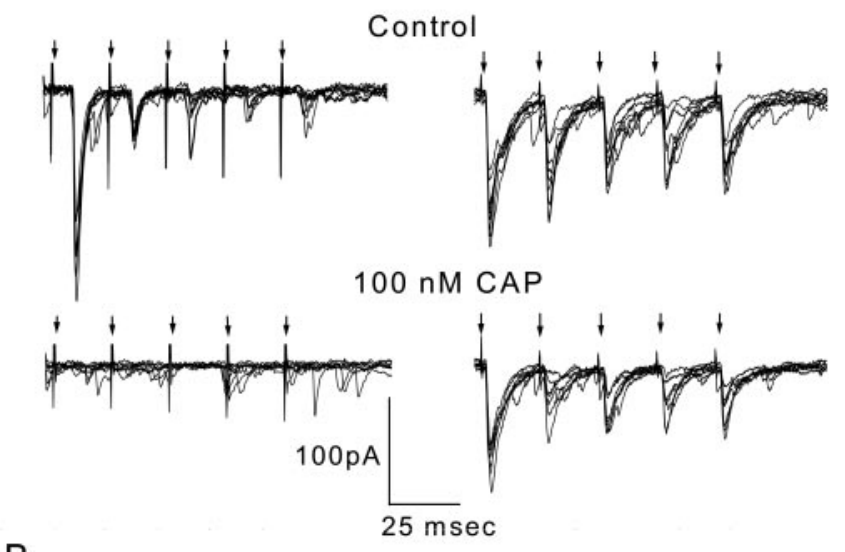

B
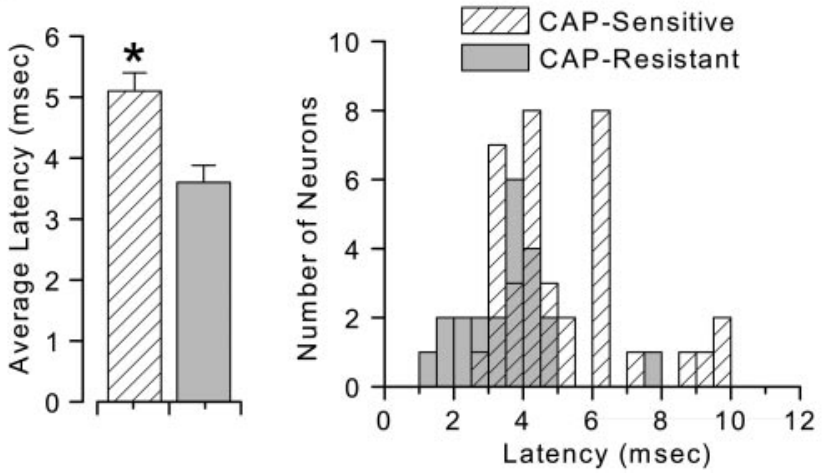

Figure 1. CAP sensitivity of ST-evoked synaptic responses in mNTS neurons. ST stimulation [five shocks (arrows) at $20 \mathrm{msec}$ intervals] consistently triggered EPSCs in these mNTS neurons. Neurons with synaptic jitters (SD of latency) of $<150 \mu \mathrm{sec}$ were selected for study and considered to be second-order mNTS neurons. The holding potential was -70 $\mathrm{mV}$. The sustained application of CAP (100 nM) blocked ST-evoked EPSCs in CAP-sensitive neurons but not in the remaining neurons (CAP-resistant). A, Left, Nine successive ST-evoked EPSCs from one such CAP-sensitive neuron before (top) and $5 \mathrm{~min}$ after (bottom) CAP application. $A$, Right, ST EPSCs from a representative CAP-resistant neuron before (top) and $5 \mathrm{~min}$ after (bottom) CAP application. B, Left, Average latency of ST-evoked EPSCs in CAP-sensitive neurons (hatched bar; $n=37$ ) was significantly longer than in CAP-resistant neurons (solid bar; $n=22 ; * p=0.0016$; Student's $t$ test). B, Right, Histogram (bin size, $0.5 \mathrm{msec}$ ) of the distribution for these individual latencies in CAPresistant (solid bars) and CAP-sensitive (hatched bars) neurons. Although group average latencies were different, the two overlapped considerably.

sient currents in CAP-sensitive neurons (Fig. 2B, top). These early transient, voltage-dependent currents resemble A-type potassium currents (Pongs, 1999). In contrast, outward currents in CAP-resistant neurons were dominated by sustained currents with little early transient component (Fig. $2 A, B$, middle). Average peak SSCs (Fig. 3) were significantly different in CAP-sensitive compared with CAP-resistant neurons $(p<0.0001$; RM ANOVA). At activation voltages from -60 to $0 \mathrm{mV}$, the average TOCs were significantly larger in CAP-sensitive neurons compared with those observed in CAP-resistant neurons $(p<0.0011$; one-way ANOVA and Fisher's PLSD). The SS component of these currents in the same neurons was indistinguishable between CAP-sensitive and CAP-resistant neurons $(p=0.752 ; \mathrm{RM}$ ANOVA) (Fig. 3B). The outward current patterns were highly dependent on the membrane potential preceding the depolarization step. From hyperpolarized holding potentials $(-90 \mathrm{mV})$, 

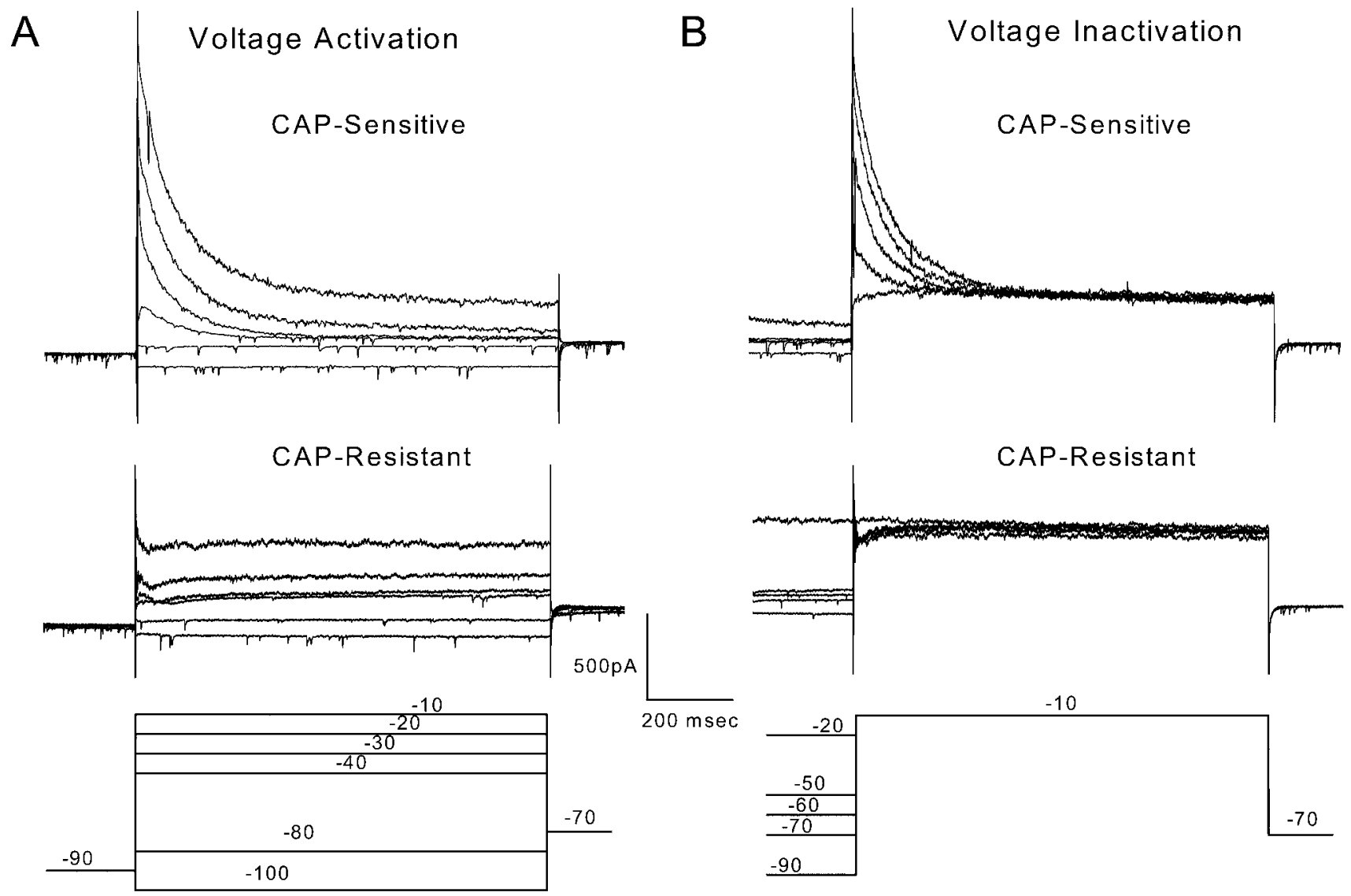

$200 \mathrm{msec}$

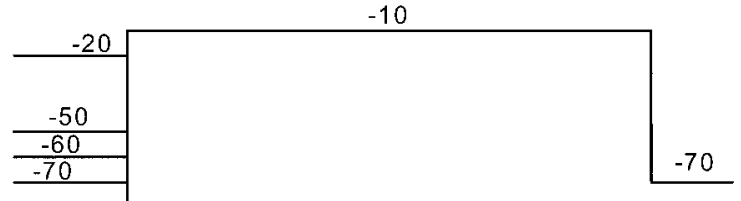

Figure 2. Voltage-dependent activation and inactivation of outward currents in two representative second-order mNTS neurons, one CAP-sensitive and one CAP-resistant. $A$, Prolonged depolarization evoked prominent TOCs in CAP-sensitive neurons but not in CAP-resistant neurons. All neurons were held at $-70 \mathrm{mV}$ before each test sequence. Neurons were preconditioned at $-90 \mathrm{mV}$ for $500 \mathrm{msec}$ followed by step commands to test potentials ( -100 to $0 \mathrm{mV}$ in $10 \mathrm{mV}$ increments). For clarity, only six representative current responses $(A$, bottom $)$ are displayed. The large outward current to sustained depolarization of the CAP-sensitive neuron (top) rapidly decayed. Current traces from a representative CAP-resistant neuron (middle) show little such TOC. B, Conditioning neurons at depolarized potentials suppressed the TOC component in the CAP-sensitive neuron (top) but had little effect on the outward currents of CAP-resistant (middle) neurons (representative neurons). Whole-cell currents were measured in response to step voltage commands. Between sweeps, neurons were held at $-70 \mathrm{mV}$. Conditioning steps varied in $10 \mathrm{mV}$ increments from -100 to $0 \mathrm{mV}$. Conditioning was followed by a prolonged $(1200 \mathrm{msec})$ step to $-10 \mathrm{mV}$. For clarity, only 5 of the 11 sweeps are shown. Voltage step protocols are outlined in the bottom panels.

depolarizing steps activated TOCs beginning at more than -60 $\mathrm{mV}$ (Fig. 3A). These TOCs had similar voltage-dependent activation in both CAP-sensitive and CAP-resistant neurons (Fig. $3 A$ ). CAP-resistant neurons generally had the smallest peak transient currents, whereas CAP-sensitive neurons had the largest (Fig. $3 C$ ). However, the two groups overlap in the magnitude of peak TOCs (Fig. 3C). Inactivation of the normalized TOC in CAP-sensitive neurons (Fig. 4) was steeply dependent on the voltage in the range nearest the resting potential from -70 to -50 $\mathrm{mV}$. Together, these results suggest that second-order neurons connected to VR1-containing afferents express larger TOCs than neurons connected to afferents without VR1. The TOCs in these mNTS neurons resemble A-type $\mathrm{K}^{+}$currents $I_{\mathrm{KA}}$ (Dekin and Getting, 1987; Moak and Kunze, 1993).

\section{Pharmacological characterization of outward currents} 4-AP reliably blocks A-type $\mathrm{K}^{+}$currents (Pongs, 1999) and identified $I_{\mathrm{KA}}$ in dissociated NTS neurons (Moak and Kunze, 1993). In subsets of our CAP-sensitive mNTS neurons, we tested whether the TOCs or SSC outward currents were sensitive to 4-AP or TEA. 4-AP selectively depressed the TOC in a concentration-dependent manner $(p=0.0001)$ without altering the sustained, steady-state level of the current (Fig. $5 \mathrm{~A}$, top right).
In the voltage range for activation ( -40 to $0 \mathrm{mV}$ ), both 1 and 5 mM 4-AP consistently reduced the group average peak TOC (Fig. $5 A$, top right $)$ compared with controls $(p<0.026)$. 4-AP did not significantly affect SSCs $(p=0.262)$ (Fig. $5 A$, bottom right). TEA $(10 \mathrm{~mm})$, in contrast, depressed only the $\operatorname{SSC}(p=0.0014)$ without altering the TOCs $(p=0.663)$ (Fig. $5 B)$. Application of TEA reduced SSCs at -10 and $0 \mathrm{mV}(p<0.0007)$ (Fig. $5 B$, right). Together, these results identify the TOC as the 4-APsensitive $I_{\mathrm{KA}}$; this current appears to be expressed much more in second-order mNTS neurons with CAP-sensitive afferent inputs.

\section{$\mathrm{K}^{+}$current differentially modulates synaptic excitation in CAP-sensitive and CAP-resistant mNTS neurons}

Such substantial differences in $\mathrm{K}^{+}$currents between CAPsensitive and CAP-resistant mNTS neurons should have an important impact on the ability of afferent synaptic inputs to excite the postsynaptic neurons and, therefore, to determine the characteristics of the sensory throughput of these pathways. To assess this, we measured responses to current injection (Fig. 6) and to ST activation (Fig. 7) under current clamp in representative CAP-sensitive and CAP-resistant second-order neurons. Equivalent hyperpolarizing current injection evoked a delayed excitation in CAP-sensitive neurons to subsequent depolarizing current 

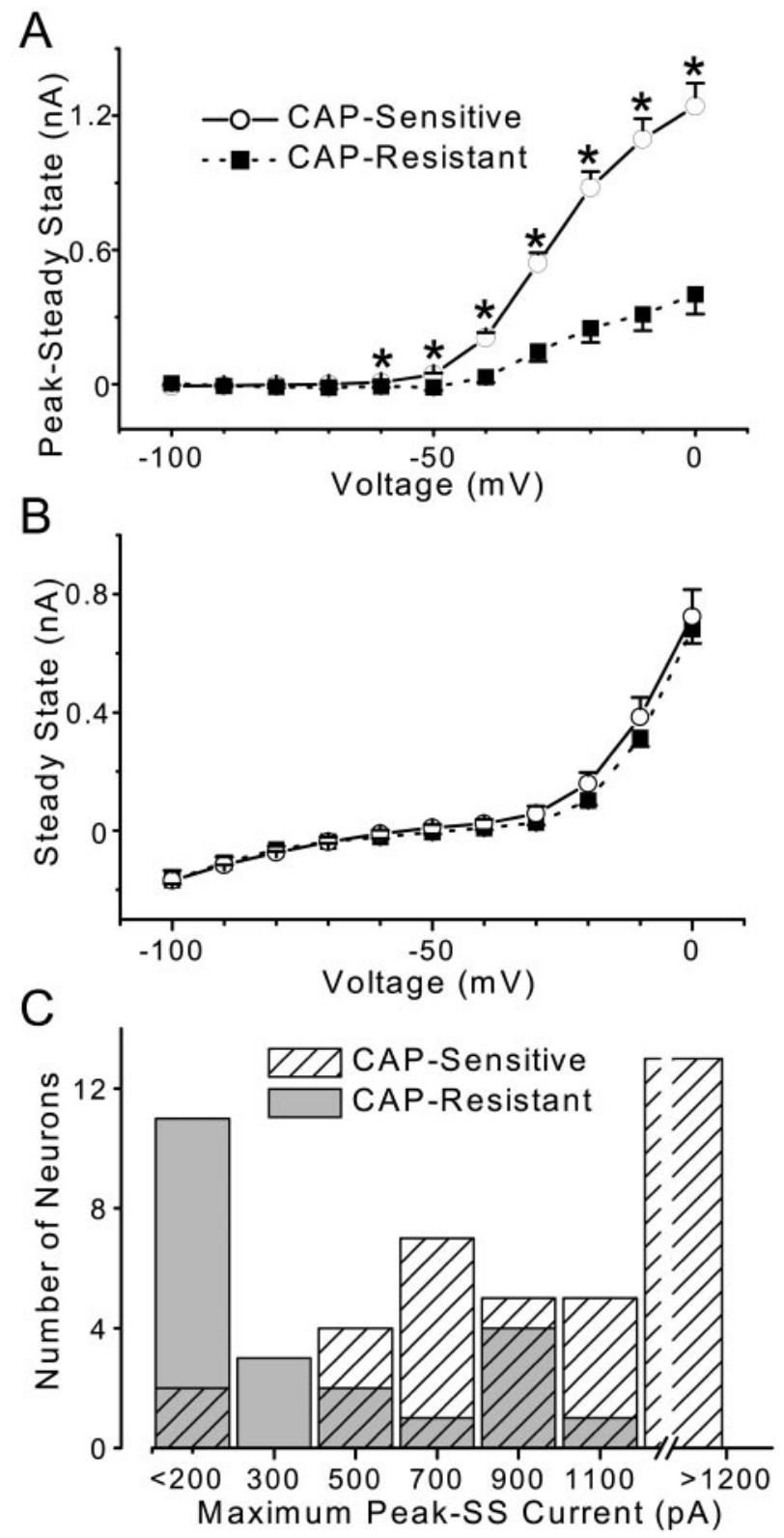

Figure 3. Summary of average outward currents in CAP-sensitive $(n=$ $37)$ and CAP-resistant $(n=22)$ mNTS neurons. Voltage-dependent TOCs (Peak - SS) and SSCs. Voltage step protocols were identical to those in Figure 2. Points are averages \pm SEM. A, CAP-sensitive neurons (solid line) had a significantly larger TOC compared with CAP-resistant neurons (dashed line; $p<0.0001 ; \mathrm{RM}$ ANOVA). Comparison of the TOC observed at specific voltages revealed that TOCs were larger in CAPsensitive neurons beginning at $-60 \mathrm{mV}$ and extending to $0 \mathrm{mV}$ (one-way ANOVA; $* p<0.001)$. $B$, Average SSCs were similar $(p=0.752$; RM ANOVA) in CAP-sensitive (solid line) and CAP-resistant (dashed line) neurons. $C$, Distribution of peak TOCs in CAP-sensitive and CAPresistant mNTS second-order neurons. CAP-sensitive neurons (hatched bars) generally expressed larger TOCs than did CAP-resistant neurons (solid bars), although there is some overlap.

injection (Fig. 6B, top, arrow), and this was not present in CAPresistant neurons (Fig. 6B, middle). The length of this delay was modulated by both the magnitude and the duration of membrane hyperpolarization in a manner consistent with the activation of $I_{\mathrm{KA}}$ (data not shown). In CAP-sensitive second-order neurons, the generation of action potentials during synaptic activation

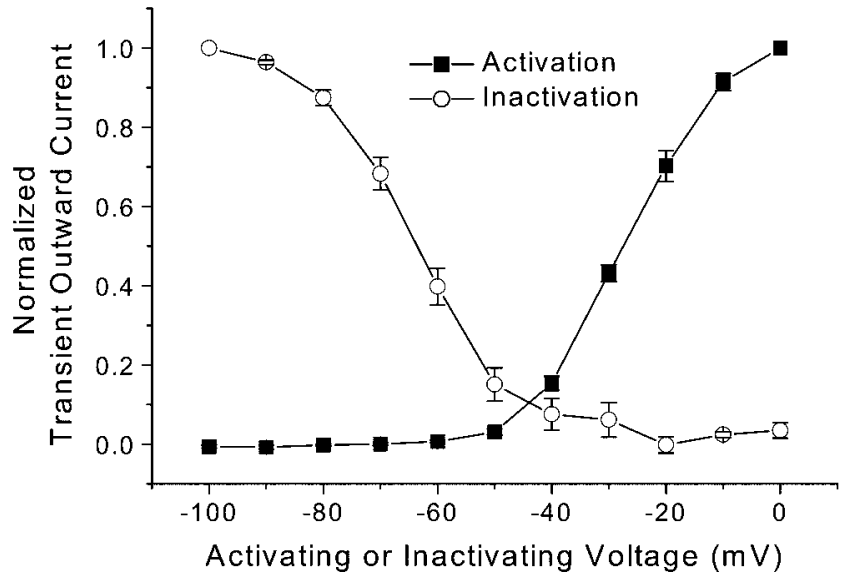

Figure 4. Summary of mean activation and inactivation relationships for TOCs of CAP-sensitive neurons. Voltage protocols were identical to those in Figure 2. Average normalized peak TOCs \pm SEM $(n=37)$ are plotted against activation voltage $(\square)$ or conditioning voltage $(\bigcirc)$. These voltage-dependent activation and inactivation characteristics of TOCs resemble those reported for A-type, 4-AP-sensitive potassium currents.

should be quite dependent on membrane potential history. ST stimulation reliably evoked action potentials in these neurons at steady resting potentials (Fig. 7A, left). However, when preceded by significant membrane hyperpolarization, the same ST stimuli often failed to produce action potentials (Fig. 7A, right). In contrast, in a CAP-resistant neuron without a notable $I_{\mathrm{KA}}$, membrane hyperpolarization did not reduce ST-evoked action potentials (Fig. 7B). Together, these results demonstrate that the differential expression of $I_{\mathrm{KA}}$ in CAP-sensitive mNTS secondorder neurons will result in very different processing of visceral afferent inputs.

\section{DISCUSSION}

\section{Second-order mNTS neurons with VR1-containing} afferents express larger A-type $\mathrm{K}^{+}$current

Our studies have identified two groups of second-order neurons within the mNTS that have very different patterns of postsynaptic expression of $\mathrm{K}^{+}$current subtypes. The sensitivity of STactivated EPSCs to block by sustained CAP exposure divided the neurons into two groups, CAP-sensitive and CAP-resistant. CAP blocks ST-evoked EPSCs by the presynaptic activation of VR1 receptors (Doyle et al., 2002). CAP-sensitive neurons had significantly larger absolute TOCs than CAP-resistant NTS neurons. The SSCs were similar in all mNTS second-order neurons. Both the voltage- and time-dependent characteristics of the TOC and the pharmacological sensitivity were consistent with A-type potassium currents, $I_{\mathrm{KA}}$. Our results demonstrate that in CAP-sensitive neurons, the activation of $I_{\mathrm{KA}}$ reduced neuronal excitability and depressed the generation of action potentials by ST-evoked synaptic transmission. This preferential expression of $I_{\mathrm{KA}}$ in VR1-containing pathways suggests that differences in presynaptic afferents are coupled with key postsynaptic differences at the second-order neuron in the mNTS.

\section{CAP discrimination of A- and C-fiber afferent inputs to the mNTS}

The NTS receives a wide range of both fast-conducting, myelinated afferents and slow-conducting, unmyelinated afferents from the viscera (Loewy, 1990; Spyer, 1990; Andresen and Kunze, 1994). Many of these afferents are well known to be activated by 

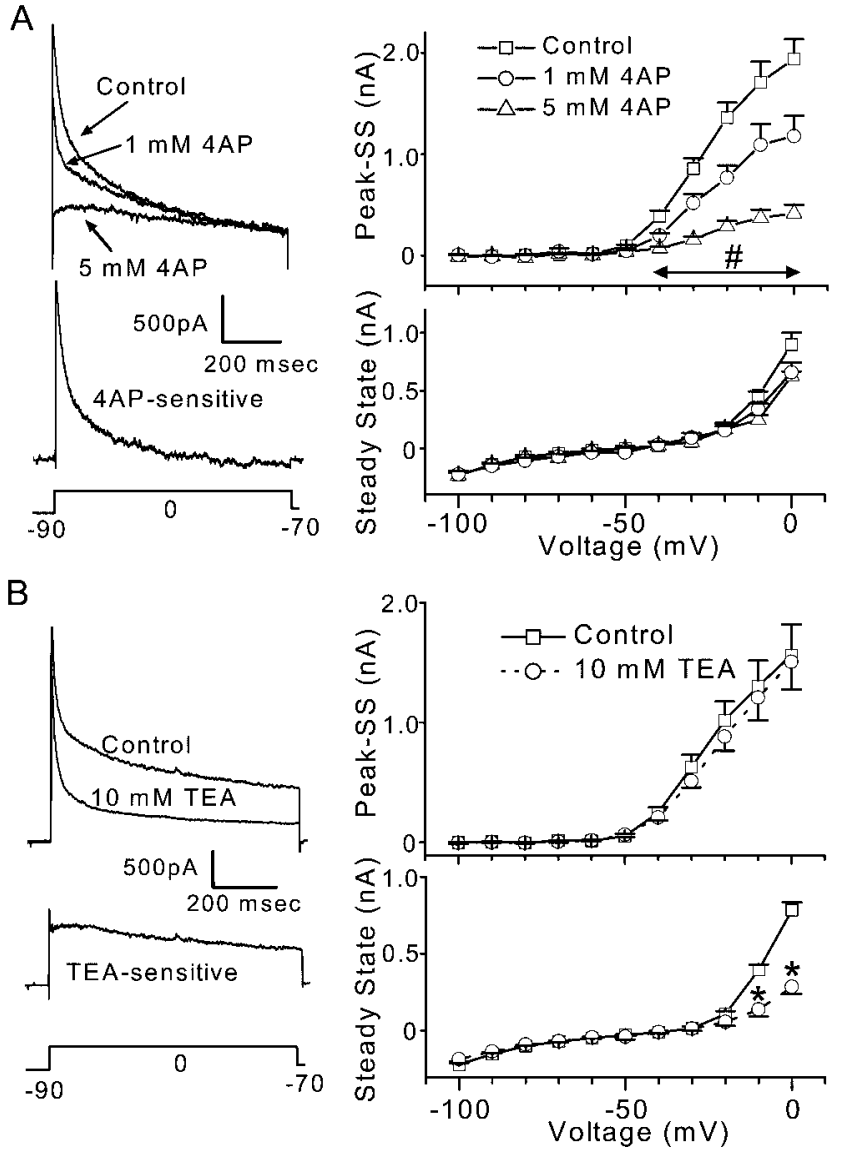

Figure 5. Pharmacological sensitivity of outward currents in CAPsensitive mNTS neurons. TOC was calculated as the peak - SSC and SSC measured at the end of the test step. Command voltage step protocols are indicated in the bottom traces of $A$ and $B$. Average values are means \pm SEM in each summary relationship. A, Left, Three current traces from a representative experiment generated by the step command in the bottom trace show the effects of 1 and $5 \mathrm{~mm}$ 4-AP on TOCs and SSCs. 4-AP reduced the TOC in this neuron in a dose-dependent manner but did not affect the SSCs. The middle trace is a difference current obtained by subtracting the control and $5 \mathrm{~mm}$ 4-AP traces to yield the net 4-AP sensitive current. Right, Summary data for 1 and $5 \mathrm{~mm}$ 4-AP on TOCs and SSCs in five CAP-sensitive neurons. Points are averages \pm SEM in controls $(\square)$, with $1 \mathrm{~mm}$ 4-AP $(\bigcirc)$, and $5 \mathrm{~mm}$ 4-AP $(\triangle)$. Both 1 and $5 \mathrm{~mm}$ 4-AP caused a significant reduction in voltage-activated TOCs compared with controls ( $p=0.0001$; RM ANOVA). Both 1 and 5 mM 4-AP reduced the TOCs observed at -40 through $0 \mathrm{mV}\left({ }^{\#} p<0.025\right.$; one-way ANOVA and Fisher's PLSD). SSCs were not affected by 4-AP (bottom right; $p=$ 0.262 ; RM ANOVA). These results suggest that the TOC observed in CAP-sensitive mNTS neurons is an A-type potassium current. B, Left, Two representative current traces evoked by steps to zero potential from $-90 \mathrm{mV}$ conditioning levels. The middle trace is a difference current obtained by subtracting the control and $10 \mathrm{~mm}$ TEA traces to yield the net TEA-sensitive current. Right, Summary data for TEA $(10 \mathrm{mM})$ in seven CAP-sensitive neurons. TEA had no effect on the TOC $(p=0.6626$; RM ANOVA) but reduced the SSCs ( $p=0.0014$; RM ANOVA) at -10 and $0 \mathrm{mV}\left({ }^{*} p<0.0007\right.$; one-way ANOVA with Fisher's PLSD).

brief exposure to the VR1 agonist CAP (Marsh et al., 1987; Bevan et al., 1992; Li and Owyang, 1996; Malinowska et al., 2001). Neonatal CAP treatment permanently and selectively eliminates C-type afferent neurons (Bevan and Szolcsányi, 1990; Holzer, 1991; Szallasi and Blumberg, 1999). Prolonged short-term application of CAP to the aortic depressor nerve, a nerve trunk that contains only aortic baroreceptor afferent axons (Sapru and Krieger, 1977; Kobayashi et al., 1999), selectively blocked the
A
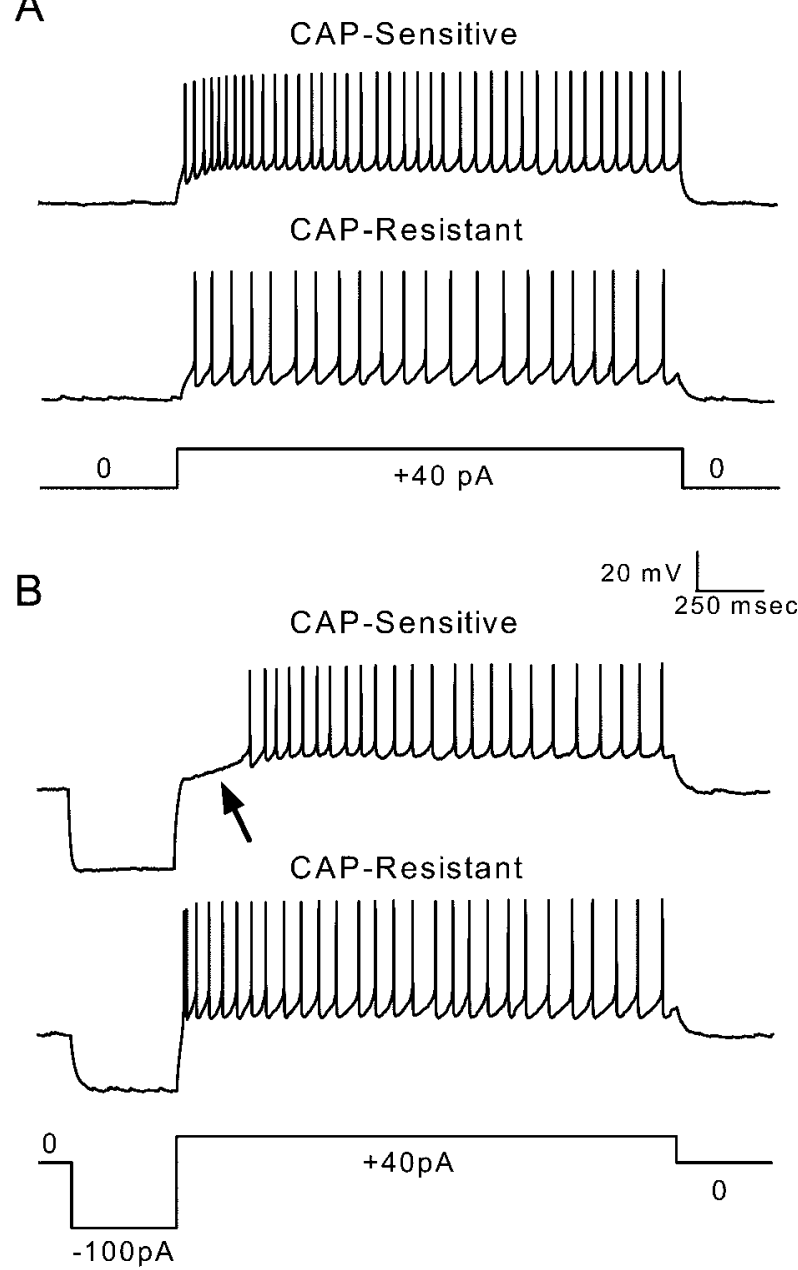

Figure 6. The differential expression of A-type potassium currents produces different firing properties in second-order CAP-sensitive and CAPresistant mNTS neurons. $A$, Representative voltage traces measured in current-clamp mode from a CAP-sensitive (toptrace) and a CAP-resistant (middle trace) neuron show evoked firing produced by current injection shown in the current command below $(+40 \mathrm{pA})$. Both neurons fire repeated action potentials with little or no delay after the onset of current injection. $B$, Representative voltage traces measured in current-clamp mode from the same CAP-sensitive (top trace) and CAP-resistant (middle trace) neurons in $A$ showing evoked firing after negative current-evoked membrane hyperpolarization and subsequent depolarization $(-100$ and $+40 \mathrm{pA}$, step current command shown in bottom trace). The hyperpolarization caused a delay in the production of current-evoked action potentials (arrow) in the CAP-sensitive neuron. In contrast, there was no delay in firing produced by hyperpolarization in the CAP-resistant neurons. The CAP-sensitive neuron from which these voltage traces were recorded displayed a large $I_{\mathrm{KA}}$-like current. The CAP-resistant neuron did not contain such a current. The delay in evoked firing was likely caused by hyperpolarization-induced removal of inactivation of A-type potassium currents.

conduction of C-type axons and their reflex effects (Fan and Andresen, 1998). In another study (Doyle et al., 2002) and this study, application of CAP to brainstem slices blocked ST-evoked EPSC transmission to second-order mNTS neurons; we conclude that this pharmacological sensitivity is consistent with the presence of VR1 on C-type but not A-type cranial visceral afferent axons. These results in ST afferent axons are consistent with the known properties of the peripheral portions of cranial visceral afferents. Thus, CAP-sensitive ST responses have longer average 

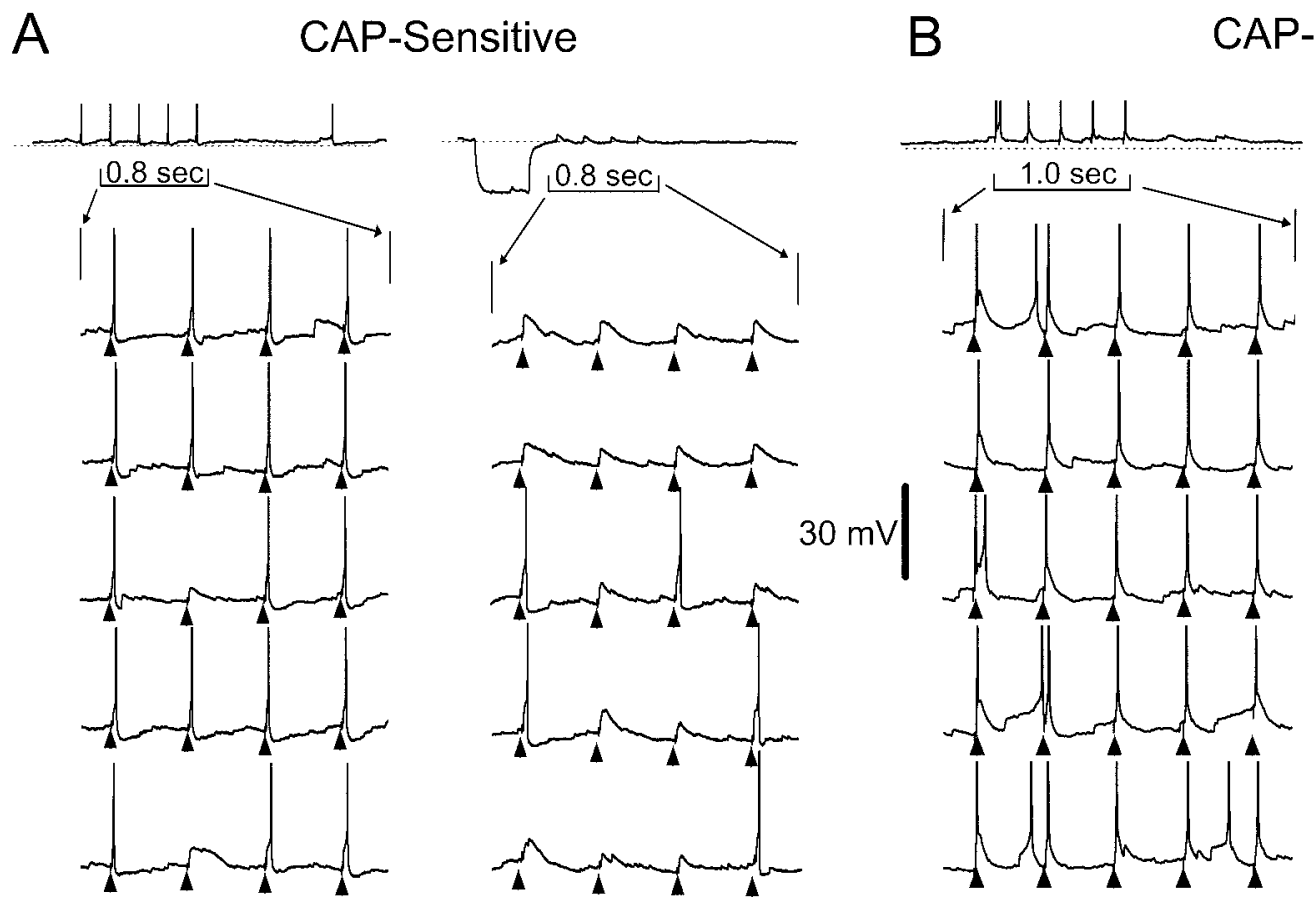

CAP-Resistant

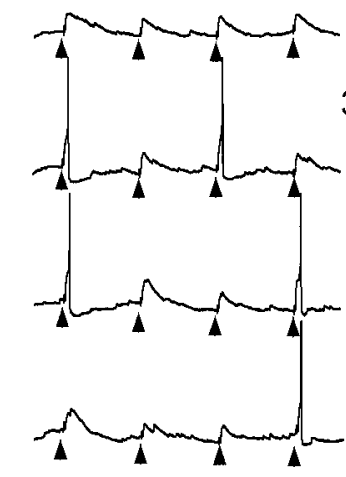

B

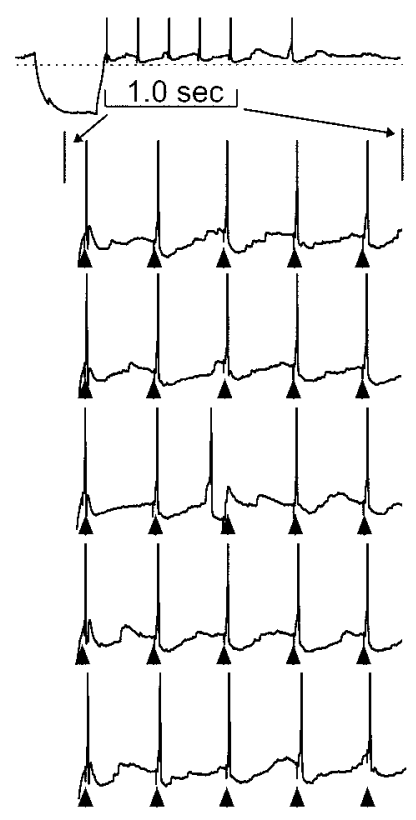

Figure 7. A-type potassium currents have an impact on the processing of ST-evoked responses in second-order mNTS neurons. $A$, Representative sequential voltage traces measured in current-clamp mode from a representative CAP-sensitive neuron (same neuron as Fig. 6) showing ST-evoked membrane potential responses. Top traces show a complete, $2.5 \mathrm{sec}$ test with ST-evoked EPSPs and action potentials. Bottom traces show expanded sweeps of five consecutive tests to better display responses to bursts of five ST shocks (triangles). Note that the voltage scaling of the expanded sweeps truncates action potentials. ST stimulation in controls produced EPSPs that reliably generated action potentials $(A$, left $)$. Only 2 of 20 ST shocks failed to trigger an action potential in this example. Introduction of a hyperpolarizing current step (top, long trace) suppressed action potentials but reliably produced EPSPs to ST shocks in this CAP-sensitive neuron $(A$, right $)$. $B$, Tests similar to those shown in $A$ were conducted on a representative CAP-resistant neuron (same neuron as Fig. 6). ST stimulation (triangles) reliably produced EPSPs that generated action potentials with and without conditioning hyperpolarization. These observations suggest that the expression of A-type currents has a differential impact on the processing of afferent inputs in CAP-sensitive and CAP-resistant second-order mNTS neurons.

latencies than CAP-resistant ST pathways. Together, our results suggest that CAP pharmacologically discriminates between Aand C-fiber inputs to second-order neurons in the mNTS.

\section{Potassium channels in sensory integration}

Potassium-selective ion channels display a wide range of gating and kinetic properties that may shape cellular integration (Coetzee et al., 1999; Pongs, 1999; Trussell, 1999; Choe, 2002), yet their contribution within the fuller context of a physiological system is poorly understood (Coetzee et al., 1999). Our present studies have identified a differentiation of afferent pathways for two pharmacological classes of visceral sensory afferents based on the presence of VR1 and their consequent CAP sensitivity. These two pathways contain second-order neurons with substantially different $\mathrm{K}^{+}$channel expression and correspond to A- and $\mathrm{C}$-fiber visceral afferent pathways. Previous studies of the intrinsic properties of NTS neurons have proposed subgroups based on morphological and electrophysiological differences, including $I_{\mathrm{KA}}$ (Dekin and Getting, 1987; Dekin et al., 1987; Moak and Kunze, 1993; Schild et al., 1993; Tell and Bradley, 1994), but their relationship to functional processing within the NTS was unknown. $I_{\mathrm{KA}}$ finely modulates the intrinsic firing rate, action potential threshold, and interspike interval (Dekin and Getting, 1987; Dekin et al., 1987; Schild et al., 1993; Pongs, 1999). Comprehensive models of the ionic currents of mNTS neurons suggest an intimate interaction of voltage and time dependence, with a key role for $I_{\mathrm{KA}}$ in the modulation of these multiple aspects of neuronal excitability (Schild et al., 1993). The removal of $I_{\mathrm{KA}}$ from model neurons yields a dichotomy of predicted performance results that are quite similar to our present results from currentclamp recordings in response to current injection. Ultimately, these findings indicate that the transduction process of afferent action potential trains into spikes in the second-order mNTS neurons will be distinctly different through these two classes (CAP-sensitive and CAP-resistant) of afferent pathways and should give rise to functionally distinct reflex responses.

\section{Divergent processing along A- and C-type afferent pathways in the $\mathrm{mNTS}$}

The voltage and time dependence of $I_{\mathrm{KA}}$ predicts a substantial impact on the processing of afferent inputs in the reflex pathways through the NTS. The respective activation and inactivation relationships for $I_{\mathrm{KA}}$ in NTS neurons were steeply dependent on membrane potential and suggest that the depolarizations and hyperpolarizations that accompany excitatory and inhibitory synaptic transmission will strongly affect $I_{\mathrm{KA}}$ activity and therefore neuronal excitability in a physiologically critical range. GABAergic inhibitory synaptic inputs are common on second-order mNTS neurons and frequently accompany afferent volleys through what appear to be local inhibitory circuits (Andresen and Yang, 1990; Mifflin and Felder, 1990; Andresen and Mendelowitz, 1996).

Our recordings were limited to the mNTS. This portion of the NTS contains the greatest density of aortic baroreceptor terminal fields (Mendelowitz et al., 1992). Selective activation of C-type afferents from aortic baroreceptors evokes reflex responses that 
are markedly different in their frequency-response relationships than for A-type aortic baroreceptors (Fan et al., 1999). These reflex responses are particularly dependent on the pattern of the afferent volley and, therefore, the temporal distribution of afferent spikes entering the CNS (Seagard et al., 1993; Fan and Andresen, 1998; Fan et al., 1999). Burst patterns of stimulation elicited greater reflex changes in blood pressure with A-type but not C-type afferents compared with constant stimulus frequencies (Fan and Andresen, 1998). Part of this frequency-dependent matching between the A-type, naturally high-frequency baroreceptor action potential trains, with burst responsiveness of A-type baroreflex responses, may well reflect a relative paucity of $I_{\mathrm{KA}}$ and the braking effects of its activation at the second-order neuron.

\section{Baroreflex modulation via A-type $\mathrm{K}^{+}$current}

An intriguing prospect of $I_{\mathrm{KA}}$ is its association with modulation by neurotransmitters (Gage, 1992; Jänig and McLachlan, 1992; Deadwyler et al., 1995; Wang et al., 1997). A wide range of neurotransmitters, including neuropeptides, are found in the NTS; many of these appear to be differentially distributed across A- and C-type afferents (Andresen and Kunze, 1994). Thus, divergent processing of $\mathrm{A}$ - and $\mathrm{C}$-fiber afferent inputs may be directed toward the modulation of $I_{\mathrm{KA}}$. Voltage-dependent $\mathrm{K}^{+}$ currents in the CNS are modulated by neurotransmitters such as substance P (Haeusler and Osterwalder, 1980), vasopressin (Oz et al., 2001), angiotensin II (Wang et al., 1997), and calcitonin gene-related peptide (Zona et al., 1991). Interestingly, our results indicate that $I_{\mathrm{KA}}$ is preferentially expressed in second-order neurons with $\mathrm{C}$-fiber afferents. This coupling of afferent fibers containing peptidergic machinery with postsynaptic neurons containing cellular targets provides a potential mechanism for the modulation of afferent signal processing through cellular targets on second-order neurons.

Overall, our results indicate that mNTS neurons that are contacted by $\mathrm{C}$-fiber cranial visceral afferents preferentially express greater $I_{\mathrm{KA}}$ compared with those connected to A fibers. This offers an intriguing bias toward the lower frequencies of transmission, with reduced fidelity to high frequencies of excitatory visceral afferent inputs in the C-type pathway. Thus, even at the first central neuron, this frequency transmission in NTS resembles the frequency performance of the full C-type pathway for certain reflexes, such as the baroreflex. This nonuniform expression of potassium channels in second-order neurons likely contributes to the divergent processing of $\mathrm{A}$ - and $\mathrm{C}$-fiber afferents in mNTS. Because of the important position of these neurons in the baroreflex loop, $I_{\mathrm{KA}}$ may represent a cellular target for modulation of the baroreflex by neurotransmitters in various physiological conditions that transform the reflex.

\section{REFERENCES}

Andresen MC, Kunze DL (1994) Nucleus tractus solitarius: gateway to neural circulatory control. Annu Rev Physiol 56:93-116.

Andresen MC, Mendelowitz D (1996) Sensory afferent neurotransmission in caudal nucleus tractus solitarius: common denominators. Chem Senses 21:387-395.

Andresen MC, Yang M (1990) Non-NMDA receptors mediate sensory afferent synaptic transmission in medial nucleus tractus solitarius. Am J Physiol 259:H1307-H1311.

Bevan S, Szolcsányi J (1990) Sensory neuron-specific actions of capsaicin: mechanisms and applications. Trends Pharmacol Sci 11:330-333.

Bevan S, Hothi S, Hughes G, James IF, Rang HP, Shah K, Walpole CS, Yeats JC (1992) Capsazepine: a competitive antagonist of the sensory neurone excitant capsaicin. Br J Pharmacol 107:544-552.
Choe S (2002) Potassium channel structures. Nat Rev Neurosci 3:115121.

Coetzee WA, Amarillo Y, Chiu J, Chow A, Lau D, McCormack T, Moreno H, Nadal MS, Ozaita A, Pountney D, Saganich M, Vega-Saenz DM, Rudy B (1999) Molecular diversity of $\mathrm{K}^{+}$channels. Ann NY Acad Sci 868:233-285.

Deadwyler SA, Hampson RE, Mu J, Whyte A, Childers S (1995) Cannabinoids modulate voltage sensitive potassium A-current in hippocampal neurons via a cAMP-dependent process. J Pharmacol Exp Ther 273:734-743.

Dekin MS, Getting PA (1984) Firing pattern of neurons in the nucleus tractus solitarius: modulation by membrane hyperpolarization. Brain Res 324:180-184.

Dekin MS, Getting PA (1987) In vitro characterization of neurons in the ventral part of the nucleus tractus solitarius. II. Ionic basis for repetitive firing patterns. J Neurophysiol 58:215.

Dekin MS, Getting PA, Johnson SM (1987) In vitro characterization of neurons in the ventral part of the nucleus tractus solitarius. I. Identification of neuronal types and repetitive firing properties. J Neurophysiol $58: 195-196$.

Doyle MW, Andresen MC (2001) Reliability of monosynaptic transmission in brain stem neurons in vitro. J Neurophysiol 85:2213-2223.

Doyle MW, Bailey TW, Jin Y-H, Andresen MC (2002) Vanilloid receptors presynaptically modulate cranial visceral afferent synaptic transmission in nucleus tractus solitarius. J Neurosci 22:8222-8229.

Fan W, Andresen MC (1998) Differential frequency-dependent reflex integration of myelinated and nonmyelinated rat aortic baroreceptors. Am J Physiol 275:H632-H640.

Fan W, Schild JH, Andresen MC (1999) Graded and dynamic reflex summation of myelinated and unmyelinated rat aortic baroreceptors. Am J Physiol 277:R748-R756.

Gage PW (1992) Activation and modulation of neuronal $\mathrm{K}^{+}$channels by GABA. Trends Neurosci 15:46-51.

Haeusler G, Osterwalder R (1980) Evidence suggesting a transmitter or neuromodulatory role for substance $\mathrm{P}$ at the first synapse of the baroreceptor reflex. Naunyn Schmiedebergs Arch Pharmacol 314:111-121.

Holzer P (1991) Capsaicin: cellular targets, mechanisms of action, and selectivity for thin sensory neurons. Pharmacol Rev 43:143-201.

Jänig W, McLachlan EM (1992) Characteristics of function-specific pathways in the sympathetic nervous system. Trends Neurosci 15:475481.

Kobayashi M, Cheng ZB, Tanaka K, Nosaka S (1999) Is the aortic depressor nerve involved in arterial chemoreflexes in rats? J Auton Nerv Syst 78:38-48.

Kunze DL, Andresen MC (1991) Arterial baroreceptors: excitation and modulation. In: Reflex control of the circulation (Zucker IH, Gilmore JP, eds), pp 141-166. Boca Raton, FL: CRC.

Li Y, Owyang C (1996) Pancreatic secretion evoked by cholecystokinin and non-cholecystokinin-dependent duodenal stimuli via vagal afferent fibres in the rat. J Physiol (Lond) 494:773-782.

Loewy AD (1990) Central autonomic pathways. In: Central regulation of autonomic functions (Loewy AD, Spyer KM, eds), pp 88-103. New York: Oxford UP.

Malin SA, Nerbonne JM (2001) Molecular heterogeneity of the voltagegated fast transient outward $\mathrm{K}^{+}$current, $\mathrm{I}(\mathrm{Af})$, in mammalian neurons. J Neurosci 21:8004-8014.

Malinowska B, Kwolek G, Gothert M (2001) Anandamide and methanandamide induce both vanilloid VR1- and cannabinoid CB1 receptor-mediated changes in heart rate and blood pressure in anaesthetized rats. Naunyn Schmiedebergs Arch Pharmacol 364:562-569.

Marsh SJ, Stansfeld CE, Brown DA, Davey R, McCarthy D (1987) The mechanism of action of capsaicin on sensory c-type neurons and their axons in vitro. Neuroscience 23:275-289.

Mendelowitz D, Yang M, Andresen MC, Kunze DL (1992) Localization and retention in vitro of fluorescently labeled aortic baroreceptor terminals on neurons from the nucleus tractus solitarius. Brain Res 581:339-343.

Mifflin SW, Felder RB (1990) Synaptic mechanisms regulating cardiovascular afferent inputs to solitary tract nucleus. Am J Physiol 259: H653-H661.

Moak JP, Kunze DL (1993) Potassium currents of neurons isolated from medial nucleus tractus solitarius. Am J Physiol 265:H1596-H1602.

Oz M, Kolaj M, Renaud LP (2001) Electrophysiological evidence for vasopressin V(1) receptors on neonatal motoneurons, premotor and other ventral horn neurons. J Neurophysiol 86:1202-1210.

Paton JFR, Foster WR, Schwaber JS (1993) Characteristic firing behavior of cell types in the cardiorespiratory region of the nucleus tractus solitarii of the rat. Brain Res 604:112-125.

Pongs O (1999) Voltage-gated potassium channels: from hyperexcitability to excitement. FEBS Lett 452:31-35.

Rudy B (1988) Diversity and ubiquity of K channels. Neuroscience 25:729-749.

Sapru HN, Krieger AJ (1977) Carotid and aortic chemoreceptor function in the rat. J Appl Physiol 42:344-348. 
Schild JH, Khushalani S, Clark JW, Andresen MC, Kunze DL, Yang M (1993) An ionic current model for neurons in the rat medial nucleus tractus solitarius receiving sensory afferent input. J Physiol (Lond) 469:341-363.

Seagard JL, Hopp FA, Drummond HA, Van Wynsberghe DM (1993) Selective contribution of two types of carotid sinus baroreceptors to the control of blood pressure. Circ Res 72:1011-1022.

Spyer KM (1990) The central nervous organization of reflex circulatory control. In: Central regulation of autonomic functions (Loewy AD, Spyer KM, eds), pp 168-188. New York: Oxford UP.

Standish A, Enquist LW, Schwaber JS (1994) Innervation of the heart and its central medullary origin defined by viral tracing. Science 263:232-234.
Szallasi A, Blumberg PM (1999) Vanilloid (capsaicin) receptors and mechanisms. Pharmacol Rev 51:159-212.

Tell F, Bradley RM (1994) Whole-cell analysis of ionic currents underlying the firing pattern of neurons in the gustatory zone of the nucleus tractus solitarii. J Neurophysiol 71:479-492.

Trussell LO (1999) Synaptic mechanisms for coding timing in auditory neurons. Annu Rev Physiol 61:477-496.

Wang DS, Sumners C, Posner P, Gelband CH (1997) A-type $\mathrm{K}^{+}$current in neurons cultured from neonatal rat hypothalamus and brain stem: modulation by angiotensin II. J Neurophysiol 78:1021-1029.

Zona C, Farini D, Palma E, Eusebi F (1991) Modulation of voltageactivated channels by calcitonin gene-related peptide in cultured rat neurones. J Physiology 433:631-643. 